\title{
Research on Power Data and Transmission Service Based on Satellite Resource Scheduling Algorithm
}

\author{
Peiguang Chen ${ }^{1}$, Zihao Tian ${ }^{1}$, Dong Wang ${ }^{1}$, Yeyang $\mathrm{Zhu}^{2}$ \\ ${ }^{1}$ State Grid Economic And Technical Research Institute Of Jilin Electric Power., LTD \\ ${ }^{2}$ University of Southern California
}

\begin{abstract}
Significant improvement has been made in communication capability with the development of satellite communication technology, which plays a vital role in the application of power coefficient collection and transmission. The technological development of power Internet of Things contributes to the satellite resource optimization scheduling algorithm so that the requirements for satellite data of different subscribers and different priorities can be met to the maximum extent in a short period of time. Given this background, this paper proposed an optimized satellite resource scheduling algorithm that integrated family eugenics with simulated annealing.
\end{abstract}

\section{PREFACE}

Nowadays, the application of private networks and public networks cannot completely solve the comprehensive coverage problem of smart grid communication service, especially the problem of communication intervention that is unable to realize in remote areas and places where typhoons and ice storms occur. As these areas have a relatively harsh communication construction environment, the construction of communication channels by using methods such as fiber optic laying is very costconsuming, and the construction quality shall be also constrained by the environmental conditions. The subsequent maintenance costs of the channels after construction shall be considered. Considering this, this paper chose to use the DCS earth orbit satellite data collection system for power data transmission. In this way, costs can be reduced, and the coverage area can be increased. Also, the system has strong environmental adaptability.

\section{COLLABORATIVE SATELLITE COMMUNICATION SYSTEM ARCHITECTURE DESIGN FOR POWER MULTI-SERVICE SCENARIOS}

\subsection{Overview of Low-Orbit Satellite System}

The low-orbit satellite communication system consists of three parts, which are space segment, ground segment, and subscriber segment. The space segment is composed of multiple satellites in orbital planes, and the collaboration of the satellites is mainly achieved through intra-orbit links and inter-satellite links. This mode is called "Satellite Constellation". Since the coverage of a satellite cannot ensure that communications are available at any place on earth, multiple satellites in orbit shall operate in a coordinated manner. This process is called "Satellite System". The satellites in operation are responsible for data processing and forwarding, and different satellite systems will vary due to the difference in satellite capacities. When the satellites are connected, the required network topology and routing can be built according to the satellite systems, as shown in the figure. ${ }^{[1]}$

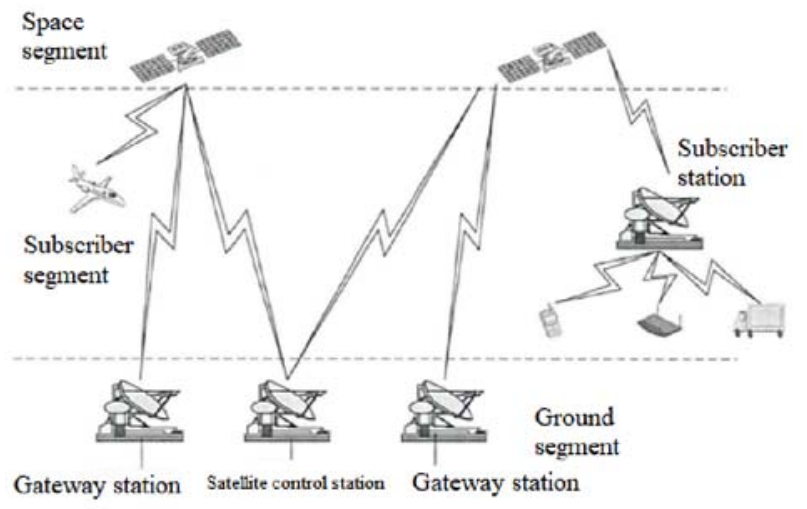

Fig 1. Low-Orbit Satellite Communication

According to the structure of the ground segment shown in the figure, the ground segment is mainly responsible for satellite network management, which is equivalent to the brain in the communication system. From a macroscopic perspective, it can control the current operation status of the satellite in real-time, and authenticate and encrypt the subscriber communication management data, and connect with the ground network. ${ }^{[2]}$ The subscriber segment is composed of several subscriber stations, including fixed subscriber

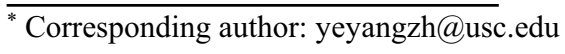


stations and mobile subscriber stations, and mobile subscriber stations can be subdivided into handheld subscriber stations, vehicle-mounted terminals, and airborne terminals.

\subsection{Location Management}

Earth orbit satellites move fast. When the position of the mobile end is the point of change of a single LEO satellite, even if the mobile terminal and beam are the same, the service time of the satellite is relatively shorter than other communication time. Earth orbit satellites can accurately transmit the data to the cellphone subscriber terminal in real-time. In this process, the change location shall be recorded, which needs to rely on the location management of satellite communications. ${ }^{[3]}$ Because the location of ground communication shall change in a consistent way, the trigger location needs to be updated in real-time, which determines the paging scheme. The location update of the earth orbit satellite communication system depends on the satellite orbit movement. Therefore, the traditional ground location management technology needs to be upgraded and transformed so as to derive a new paging strategy that can adapt to the updates of the mobile location covered by a satellite.

To better determine this strategy, an appropriate location area needs to be selected. The location area is composed of multiple cells. Subscribers can always achieve free roaming without the need of implementing location update procedures. If the subscribers are beyond the fixed boundary of the location area, an immediate update is required. The satellite system accesses the location database through a fixed earth station. ${ }^{[4]}$ In the satellite network, satellites and fixed earth stations act as the basis of the relationship among location areas. In the LEO mobile satellite network, the design of the location areas will affect the subsequent location registration and resources consumed by paging.

The figure shows the specific location management process of earth orbit satellite communication, where GS, SS, GDB, and LMB represent the gateway station, subscriber station, central database, and local database respectively. The databases can store data in location updates. Location management is the most critical part of hardware facilities. In actual application, the subscriber management process is based on the current traditional ground design scheme. When a call request is initiated, the subscriber will find the local database where the called subscriber is located according to the database and then find the current location of the called object according to the information in the database, and thus the data link is established.

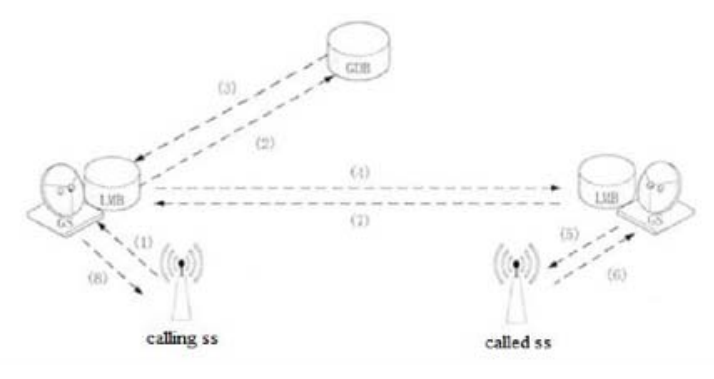

Fig 2. Calling Steps

\subsection{Switching}

Earth orbit satellites are in constant motion. Each satellite is composed of multiple beams, which cover the ground and form a single service cell. The subscriber stations in the service cell are provided by the satellite, and a beam can provide 1-2 minutes of service time at most. The subscriber will get access to new beams continuously during the call and release the resources occupied by the original beam. Even the calling time of the subscriber in the call process will be lower than the low-orbit satellite coverage time. If it is less than the subscriber's minimum elevation angle, the subscriber needs to hand it over to the visible satellite to avoid interruptions in the call. Mobile subscribers are covered by multiple satellites at any given moment, so the subscriber needs to analyze the differences between these satellites and select one out of the covered satellite as the next service satellite. No matter what kind of switching is used, its purpose is to ensure that the subscriber will not feel the discomfort caused by switching in communication. According to the number of transceivers in subscriber stations, satellites, and gateway stations, satellite switching can be divided into two modes, namely, pre-switching and soft-switching.

\subsection{Wireless Resource Management}

The wireless resource management technology enables the unified scheduling and allocation of all kinds of resources involved in the satellite communication system. It mainly makes use of the advantages of existing satellite resources to meet the requirements of subscribers in terms of service and communication quality. The management process needs to be implemented in coordination with the satellite control station and the gateway station. The existing allocable resources include carrier wave, time slot, and power. There are three types of wireless resource management methods. Pre-allocation mode is to allocate the existing resources to the object that applies for resources in advance. The dynamic allocation mode requires a portion of resources to be allocated according to the preallocation mode. ${ }^{[5]}$ When the existing resources have been allocated, new resource applications will be submitted to the corresponding network management center, which will continue to apply for resources to the terminal according to the service demand. After being used, the resources shall be released and returned. Currently, all resources are scheduled by the network 
control center in a unified way. The use of satellite wireless resources needs to be applied. During this period, the following process should be followed.

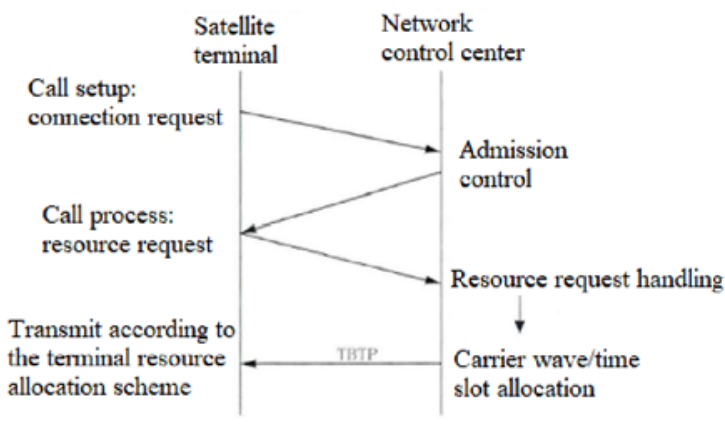

Fig. 3. Low-Orbit Satellite Wireless Resource Management System

According to the above information, this paper investigates the communication needs of the power collection network in areas without the coverage of mobile public network signals, as a way to explore the cooperative satellite system under power multi-service scenarios. Besides, a DCS satellite network data transmission system is designed for the power grid emergency and wide-area operation, maintenance, and surveillance business scenarios, as shown in the figure.

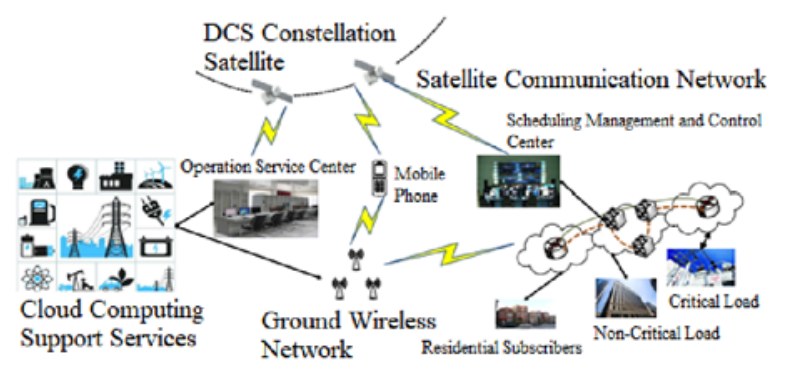

Fig 4. DCS-Based Cooperative Satellite Communication System Architecture

The system mainly includes the ground segment consisting of subscriber terminal and ground receiving and processing center and the data collection platform. The subsystems are composed of each data collection platform group and receiving stations. The central management station can be responsible for system management, including centralized regulation and control of satellite system operation, data resource interoperability, and communication distribution. The satellite communication system architecture is designed based on the above theory. With the design of a resource optimization scheduling system as the ultimate goal, the overall design framework of the satellite resource scheduling system is proposed. ${ }^{[6]}$

In this design, the resource scheduling of the ground application system is mainly to allocate the resources received and tracked, which includes equipment resources, transmission resources, and processing resources, so as to meet the current design task requirements as much as possible while making full use of the ground system resources. The system in this design can be divided into five parts, which play different roles in the system and are responsible for different tasks assigned. The main control unit module is designed for subscriber login verification and operation guidance. The satellite orbit forecast module is designed to forecast the space where the satellite is located and to send the forecast to the ground station. The resource scheduling module is designed for the scheduling, transmission, and reception of existing resources. The information management module is designed to coordinate the ground stations, satellites, and configuration information. The online module is designed to link the above-mentioned modules to realize system functions. The structure diagram is shown in Table 1 .

Table 1. Design of Satellite Resource Scheduling System Architecture

\begin{tabular}{|c|c|}
\hline \multirow{2}{*}{$\begin{array}{l}\text { Section Main } \\
\text { Control Unit } \\
\text { Module }\end{array}$} & Subscriber Login Verification \\
\hline & Operation Guidance \\
\hline \multirow{2}{*}{$\begin{array}{l}\text { Satellite } \\
\text { Orbit } \\
\text { Forecast } \\
\text { Module }\end{array}$} & Spatial Location Forecast \\
\hline & $\begin{array}{c}\text { Ground Station Track, Reception, and } \\
\text { Forecast }\end{array}$ \\
\hline \multirow{3}{*}{$\begin{array}{l}\text { Resource } \\
\text { Scheduling } \\
\text { Module }\end{array}$} & Scheduling Planning and Design Module \\
\hline & Receiving Resource Scheduling Module \\
\hline & $\begin{array}{c}\text { Transmission Resource Scheduling } \\
\text { Module }\end{array}$ \\
\hline \multirow{3}{*}{$\begin{array}{l}\text { Information } \\
\text { Management } \\
\text { Module }\end{array}$} & $\begin{array}{c}\text { Ground Station Information Management } \\
\text { Module }\end{array}$ \\
\hline & Satellite Information Management Module \\
\hline & $\begin{array}{l}\text { Configuration Information Management } \\
\text { Module }\end{array}$ \\
\hline \multirow{3}{*}{$\begin{array}{l}\text { On-line Help } \\
\text { Modul }\end{array}$} & System Overview \\
\hline & System Function Description \\
\hline & System Instruction \\
\hline
\end{tabular}

\section{Analysis and Selection of Satellite Resource Optimization Scheduling Algorithm for Power Business}

With the introduction of the concept of energy Internet and its comprehensive development, smart power distribution, distributed new energy resources, and rural network transformation have been steadily promoted. Owing to it, the current power business such as power data refinement and network distribution automation are facing new challenges, especially video business and emergency scheduling as well as precise load control for remote areas. All of these have put forward a high 
requirement for the existing bandwidth and the reliability of the application needs to be increased and the delay needs to be shortened. Besides, due to the increase in the number of nodes and coverage in existing distributed power monitoring, resource optimization scheduling has become very challenging. ${ }^{[7]}$

The current intelligent technology search method is continuously optimized and upgraded on the basis of the original. With the scheduling method as the starting point, the optimized part is to achieve intelligent operation by means of numerical calculation. In the intelligent scheduling method, the system statistical characteristics can be obtained according to the calculation. Then the loopholes that exist can be found and the optimization is realized. Such an approach can provide a new research path for optimization. However, there are also some obvious loopholes even in the proposed intelligent scheduling method. It is because, to some extent, it takes a long time to obtain the optimal solution. According to the existing research on satellite resource scheduling in China and abroad, the methods of seeking the optimal solution can be divided into several categories. In this paper, only some of the most commonly used methods are explained.

\subsection{Genetic Algorithm}

The genetic algorithm (GA) is an evolutionary algorithm. It mainly imitates Darwin's theory of evolution and uses natural selection and genetic inheritance of various living organisms as the basis to find the optimal solution. Through selection, crossover, and mutation operations, new solutions can be generated. Based on the calculation of fitness, the current individual performance can be evaluated. The population shall evolve in the direction of some function with a higher value. It will evolve in this way in the long run until the termination condition is reached. The current genetic algorithm has the advantage of random reduction and high convergence efficiency, but there will be a lot of redundancy in the solutionseeking process, and the final solution obtained is likely to be a local optimum. ${ }^{[8]}$

\subsection{Hill Climbing Method}

For the hill climbing method (HC), a solution shall be selected from the domain solution in each iteration, and the selected solution condition can make the minimum $\mathrm{f}$. Currently, there is an improved version of the hill climbing method, which is mainly to select a different initial solution in each iteration, and then use it as the starting point to achieve optimization through repeated calculations. By means of this method, a better solution can be obtained. This method is now called the "Loop Iterative Hill Climbing Method". This method can improve the quality of existing solutions, but it still cannot completely solve the defect of local optimization, which cannot be solved by using the loop iterative hill climbing method.

\subsection{Simulated Annealing Algorithm}

The simulated annealing algorithm (SA) is to achieve search optimization by simulating the high-temperature annealing process using the Metropolis acceptance criterion, so as to obtain the (approximate) global optimal solution. The objective function can be used as the energy function to control parameter temperature. The solution space can be used as the state space of the function. Under this condition, the SA algorithm is used for parameter optimization, which is also to find the minimal value of the objective function in the solution space.

With the development of the power grid business, the data communication volume is increasing drastically every day, which brings a great challenge to the security, transmission efficiency, and network resource scheduling management ability of circuit wireless communication. In order to meet the requirement for power satellite communication business and high network adaptability, it is necessary to choose a suitable resource scheduling method and then optimize the current satellite resources through having a good understanding of and comparing the advantages and disadvantages of various scheduling methods. Since the intelligent scheduling method takes a long time to obtain the optimal solution by convergence under normal operation conditions, and the implementation efficiency is low, it is difficult to judge whether the final solution obtained in the process is the optimal solution. In this paper, the simulated annealing algorithm without constraints is selected for resource scheduling of the satellite communication system to accelerate the convergence speed. In addition, the family eugenics evolution method is introduced in the research center to achieve multi-spatial target resource scheduling in the end.

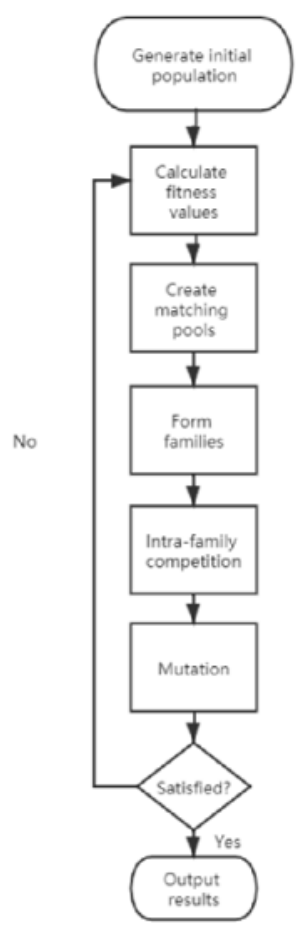

Fig. 5. Family Eugenics Algorithm Flow 


\section{Design of Satellite Resource Scheduling Scheme Based on Family Eugenics and Simulated Annealing}

\subsection{Design Objective}

In order to improve the reliability of power system communication, realize efficient data transmission, achieve the final effect of "comprehensive coverage, full collection, and complete detection", and improve the utilization rate and stability of low-orbit satellite resources, a satellite resource optimization scheduling scheme based on simulated annealing algorithm and family eugenics algorithm is innovatively proposed in this paper. The use of the family eugenics algorithm can effectively solve the defects exposed by the genetic algorithm. The key of the family eugenics algorithm is the introduction of orthogonal crossover operators. Its role is to expand the existing search range and improve search efficiency. The benefit of the family eugenics algorithm is that the individual behavior ability can be improved so that the population dispersal is strengthened. In this way, the population cannot easily be distributed to the local optimization region, so as to avoid the phenomenon of premature. Also, the convergence speed at the later stage of evolution can be accelerated. The SA algorithm is mainly to accept the deteriorated solution with a certain probability and jump out of the local optimization trap by taking the solid material degradation process as the basis, considering the similarity between the combined optimization problem, and using the Metropolis criterion. Currently, all convergence time is slow, affecting operational efficiency. ${ }^{[9]}$

\subsection{Design Scheme}

According to the design objective, the FEA-SA algorithm is specifically selected. This algorithm makes up for the shortcomings of the existing algorithm, thus improving the solution-seeking efficiency. The specific implementation plan is as follows.

Define the rate of change of PCLRB during the scheduling cycle, that is, the value of system information increment as:

$$
\Delta \mathrm{I}=\frac{\sum_{i=1, t=\Delta T_{\text {schedule }}}^{M} \Delta P C R L B_{p o s}^{i}}{\sum_{i=1, t=T_{\text {schedule }}^{\mathrm{o}} \Delta P C R L B_{p o s}^{i}}^{M} \Delta P C}
$$

Where $M$ represents the number of targets to be tracked in the scheduling cycle, the denominator term is the PCRLB sum of the location components of all tracked targets at the beginning of the current scheduling cycle, and the numerator term is the sum of the change of PCRLB of the location components of the tracked targets in the congestion cycle.
The satellite sensor switching rate is calculated as follows.

$$
S S=1-\frac{\sum_{i=1}^{M} \text { switch }_{i}}{2 \sum_{i=1}^{M} N i}
$$

Where Switch $_{i}$ is the number of times the first $i$ tracking satellite changes the tracking target in the whole scheduling cycle.

A cycle scheduling optimization model is established as follows.

$$
\begin{gathered}
\max f=\omega_{1} \Delta I+\omega_{2} S S \\
\text { s.t } \omega_{1}+\omega_{2}=1 \\
\left(x_{i}^{k}\right)_{j}, X_{i}^{j} \in\{0,1\} \\
\left(x_{i}^{k}\right)_{j}, X_{i}^{j} \in\{0,1\} \\
\left(x_{i}^{k}\right)_{j}=0, \quad \text { if } \quad S_{k} \notin S_{i}^{j} \\
\sum_{i=1}^{M} \sum_{j=1}^{N_{I}}\left(x_{i}^{k}\right)_{j}^{j} \leq 1 \\
x_{i}^{j}=1, \text { if } \sum_{k=1}^{S_{T}}\left(x_{i}^{k}\right)_{j}^{j}=2
\end{gathered}
$$

The above model depicts the sensor availability constraint and the minimum observation requirement constraint. At the same time, only one of the sensors can be assigned to a task and two satellites need to be observed within one target observation time.

For the resource scheduling strategy used in this algorithm, the specific calculation process is as follows.

(1) Establish the satellite resource set $\mathrm{S}$ and the target set $\mathrm{T}$

(2) Establish the target observation sub-task data set in the scheduling cycle

(3) Forecast the target and satellite trajectory. According to various constraints, the target observation sub-task set and its corresponding resource number set can be built. For the setup of the resource set, uniform division of inter-class time period with the arc segment method is required, as shown in Figure 6. 


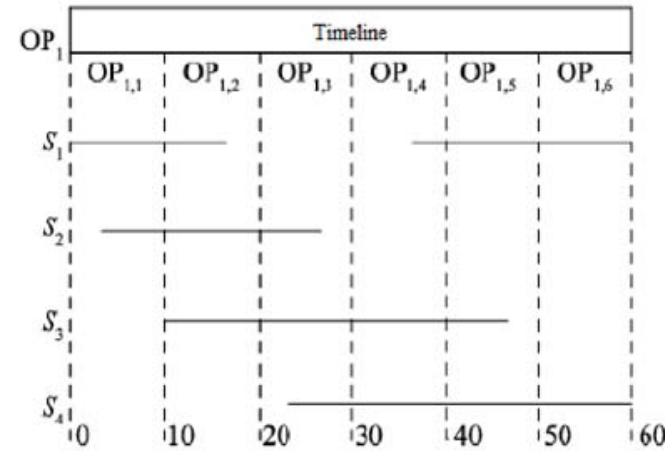

Fig. 6. Target Observation Subtask Division

When the sensor resources do not meet the requirement within the sub-task time period and the satellite observation time, if the satellite S2 within $[0,10]$ serial port is selected, it will be discarded when the visible duration does not reach half of the task time arc, otherwise, it will continue to be used as pseudo-sensor resources in the task. In the use of resources, the single satellite filtering method shall be used for target tracking in the invisible time period.

(4) The FEA-SA optimization algorithm is executed to compute the scheduling decision. The scheduling cycle can be derived based on the warning time so as to perform the execution of the scheduling decision to realize the target tracking.

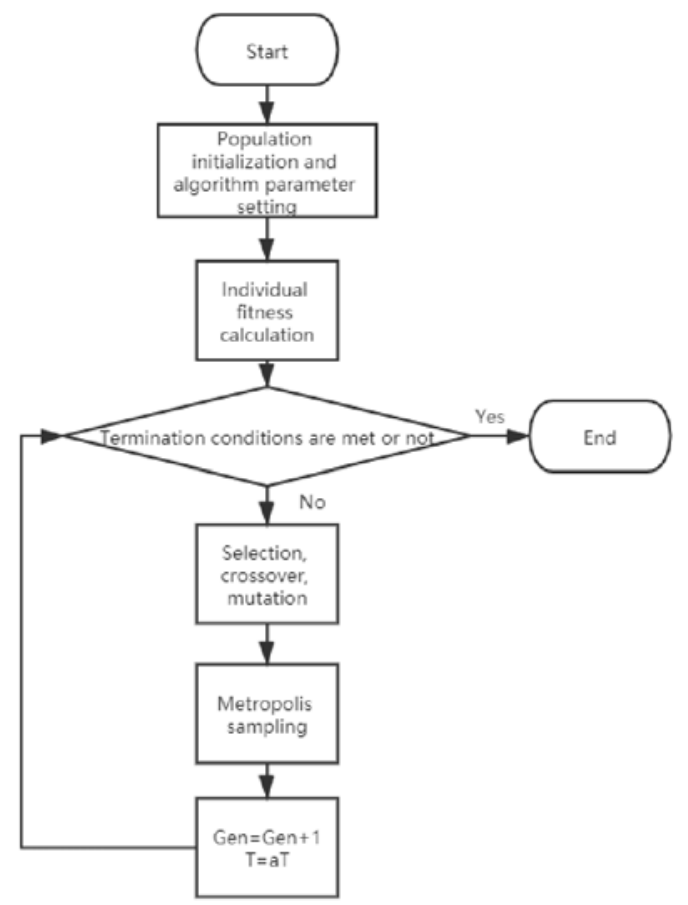

Fig. 7. FEA-SA Algorithm

\section{Conclusion}

In this paper, based on the requirements for smart grid data collection, the conflict and low resource utilization problems that occurred in the existing multi-spatial target satellite resource scheduling were solved. In particular, this paper proposed to use the family eugenics algorithm to improve the global search performance and the simulated annealing algorithm without the initial condition constraint to solve the multi-spatial target and parameter optimization problem. By doing so, the efficiency of satellite resource scheduling can be further improved, and thus high stability and high resource utilization rate of low-orbit satellite communication can be achieved.

\section{References}

1. Planet Labs Inc.; Patent Issued for Satellite Scheduling System (USPTO 10, 762, 458)[J]. Computers Networks \&amp; Communications, 2020.

2. Guansheng Peng,Guopeng Song,Lining Xing,Aldy Gunawan,Pieter Vansteenwegen. An Exact Algorithm for Agile Earth Observation Satellite Scheduling with Time-Dependent Profits[J]. Computers and Operations Research,2020,120.

3. Mingwei Yin,Jingyang Li,Xianyu Wang,Hexi Baoyin. A rapid method for validation and visualization of agile Earth-observation satellites scheduling[J]. Astrodynamics,2018,2(4).)

4. Yingjie $\mathrm{Xu}, \mathrm{Xiaolu}$ Liu,Renjie He, Yingguo Chen,Yuning Chen. Multi-objective Satellite Scheduling Approach for Very Large Areal Observation[J]. IOP Conference Series: Materials Science and Engineering,2018,435(1).

5. Xiaonan Niu,Hong Tang,Lixin Wu. Satellite scheduling of large areal tasks for rapid response to natural disaster using a multi-objective genetic algorithm[J]. International Journal of Disaster Risk Reduction,2018,28.

6. Xiaogeng Chu,Yuning Chen,Lining Xing,Filippo Cacace. A Branch and Bound Algorithm for Agile Earth Observation Satellite Scheduling[J]. Discrete Dynamics in Nature and Society,2017,2017.

7. Peng Gao,Yue Jin Tan. Multi Satellites Scheduling Problem for Area Target Based on Grid[J]. Advanced Materials Research,2013,2649.

8. Fatos Xhafa,Junzi Sun,Admir Barolli,Alexander Biberaj,Leonard Barolli. Genetic algorithms for satellite scheduling problems[J]. Mobile Information Systems, 2012,8(4).

9. Kai-Jian Zhu,Jun-Feng Li,He-Xi Baoyin. Satellite scheduling considering maximum observation coverage time and minimum orbital transfer fuel cost[J]. Acta Astronautica,2009,66(1). 